$\underline{\text { Original Article }}$

\title{
Automated Hematology Analyzers in Diagnosis of Plasmodium vivax Malaria: an Adjunct to Conventional Microscopy
}

Kolakkadan Hasaf Mubeen, Clement Wilfred Devadoss, Rau Aarathi Rangan, Monnappa Gitanjali, Shetty Prasanna and VP Sunitha

Department of Pathology, M.S Ramaiah Medical College \& Teaching Hospital, Bangalore. India

Correspondance to: Kolakkadan Hasaf Mubeen. hasafmubeen@gmail.com

Competing interests: The authors have declared that no competing interests exist.

Published: June 1, 2014

Received: October 21, 2013

Accepted: April 27, 2014

Citation: Mediterr J Hematol Infect Dis 2014, 6(1): e2014034, DOI: 10.4084/MJHID.2014.034

This article is available from: http://www.mjhid.org/article/view/12395

This is an Open Access article distributed under the terms of the Creative Commons Attribution License (http://creativecommons.org/licenses/by/2.0), which permits unrestricted use, distribution, and reproduction in any medium, provided the original work is properly cited.

Abstract. Malaria is one of the most pervasive parasitic diseases ever known to mankind affecting nearly 300 million people every year. The need for rapid diagnosis of malaria in tropical and subtropical malaria endemic areas is on the rise. In this study, we evaluated the usefulness of hematology autoanalyzers, Sysmex XE-2100 \& XT-2000i in the presumptive diagnosis of malaria. Our study shows that abnormalities in WBC/BASO scattergram when combined with presence of thrombocytopenia had a high sensitivity and positive predictive value in the presumptive diagnosis of Plasmodium vivax (P.vivax) malaria.

Introduction. The World Health Organization estimates that half the world's population is at risk of malaria, with an estimated 200-300 million people developing clinical malaria every year. ${ }^{1}$ According to World Malaria Report (2013)nearly half (273 million) of the high-risk population outside Africa resides in India ${ }^{2}$ Karnataka is located in the southern peninsular region of India and was once considered as a high transmission zone for malaria but due to the implementation of rigorous control measures the malaria incidence in the state has fallen significantly. However urban malaria has continued to be a problem in the cities of Bangalore and Mangalore in Karnataka is due to the migration of people from high risk rural areas.

In 2011, 24487 malaria cases were reported in the state of Karnataka of which $21842(89.19 \%)$ cases were infected by Plasmodium vivax species, indicating that P.vivax is the predominant species in this region. Demographic data from these cases reveal that age group 21-30 years was most affected. ${ }^{3}$

Light microscopy is considered as the gold standard approach in diagnosis of malaria, but it requires time and expertise. Many rapid diagnostic tests (RDT) have emerged recently to overcome these factors however these are expensive and not routinely available. ${ }^{4,5}$

As Complete Blood Count (CBC) is a baseline investigation ordered for patients with fever, there has been a growing focus on the utility of hematology analyzers in the presumptive diagnosis of malarial infection.

In the year 1999, Hancheid et al. reported the usefulness of automated hematology analyzer Cell Dyn 3500 (Abbott, Santa Clara, CA) in diagnosis of malaria even in the absence of clinical suspicion. ${ }^{6}$ 
This study was conducted to evaluate the utility of automated hematology analyzers, Sysmex XE-2100 \& XT-2000i (Kobe, Japan) in the diagnosis of the malarial parasite in conjunction with peripheral smear examination, by evaluating various scattergram abnormalities.

Materials and Methods. Routine blood samples from both outpatient and inpatient departments were analyzed during March 2013 to August 2013. Samples were collected in K2 EDTA tubes (Becton Dickinson, USA) and complete blood count analysis was done either on Sysmex XE-2100 or XT-2000i.Peripheral smear examination was done for all the cases.

Both analyzers use flow cytometry using a semiconductor laser to categorize WBCs based on the forward and side scattered light information of cells. Forward scattered light analyses the size and the side scattered light analyses the granularity of the WBCs. This data is depicted on coloured depictions namely WBC-DIFF and WBC/BASO scattergrams.

WBC-DIFF scattergram (WBC 4-part differential): RBCs are completely lysed with lysing solution "STROMATOLYSER-4DL", at the same time this reagent acts on WBC membranes and makes them partially permeable. Following this, a fluorescent dyeing solution "STROMATOLYSER-4DS" is added to allow the fluorescent dye to enter the WBC through its permeable membranes and stain the DNA and RNA. Following this reaction, the inbuilt flow cytometer detects the forward and side scatter information based on which WBC-DIFF scattergram is obtained. By analyzing this scattergram, the analyzer gives a 4 part differential count, viz lymphocytes, monocytes, eosinophils and other granulocytes (neutrophils plus basophils).

WBC/BASO scattergram: This scattergram is obtained by lysing RBCs with a lysing reagent

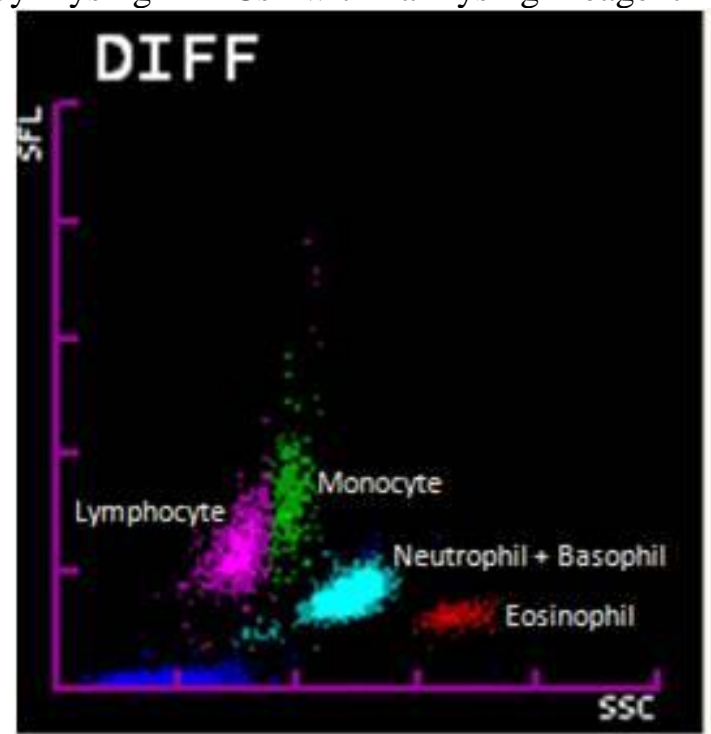

"STROMATOLYSER-FB" which selectively suppresses degranulation of basophils. Following this reaction, the sample is analysed by flow cytometry to detect forward and side scattered light information to give a WBC/BASO scattergram. The analyzer gives a total WBC count and basophil count based on this scattergram. ${ }^{8}$ Figure 1 shows a normal WBC-DIFF \& WBC/BASO scattergram.

Various changes in the WBC scattergram (WBCDIFF \& WBC/BASO)like graying of WBC clusters, merging of clusters, multiple clustering, abnormal blue coded events and other changes if any, were analyzed simultaneously for all these cases.

Hematological parameters like hemoglobin $(\mathrm{Hb})$, total leucocyte count (TLC), differential count (DC) and platelet count of all the malaria positive cases were collected.

Smears were reviewed for all the cases which showed abnormal scattergram. Sensitivity, specificity, Positive Predictive Value (PPV) and NegativePredictive Value (NPV) was calculated for both WBC-DIFF \& WBC/BASO scattergrams using Galen and Gambino method.

Results. During March 2013 to August 2013, we received 2610 peripheral smears for malarial parasite detection from patients who presented with fever, chills and rigors. Other uncommon presenting symptoms were vague abdominal pain, myalgia and headache. However, 2 cases presented with symptoms of Acute Renal Failure. Of the 2610 cases, 1730 were males and 880 were females with age ranging between 10-65 years.

Out of 2610 cases, $45(n=45)$ cases were found to be malaria positive, of which 35 were males and 10 were females with age ranging from 15-65years. Forty cases were positive for Plasmodium vivax and five cases were positive for Plasmodium falciparum with a

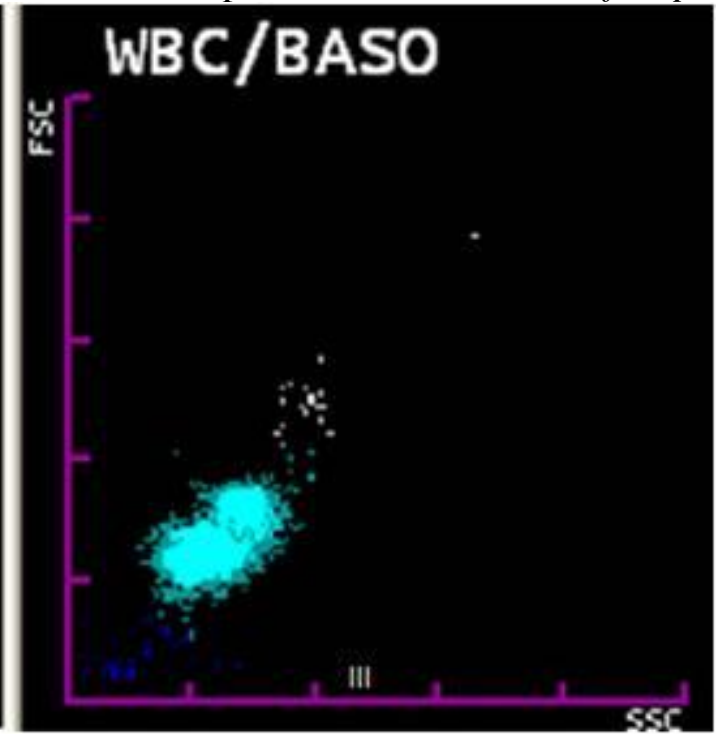

Figure 1. Normal WBC-DIFF and WBC/ BASO scattergram. Note area III in WBC/BASO scattergram. 
(Total peripheral smears)

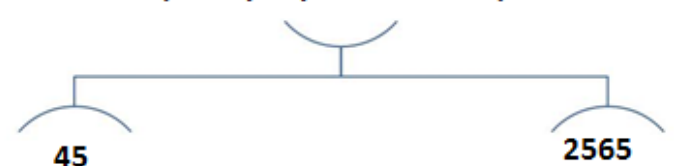

45

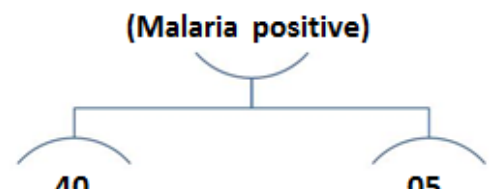

40

P. vivax

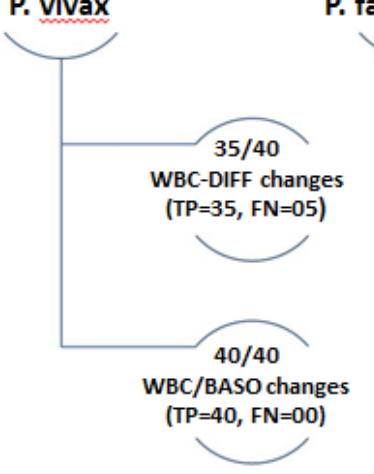

05

P. falciparum

(Malaria negative)(TN)

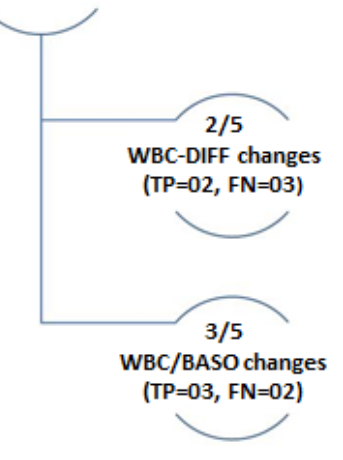

182

WBC-DIFF changes (FP)

03

WBC/BASO changes (FP)

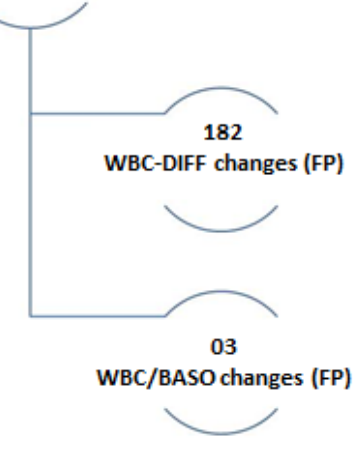

Figure 2. Distribution of cases. TP- True positive, TN- True negative, FP- False positive, FN- False negative.

P.vivax to P.falciparum ratio of 8:1.

Out of the $40 P$. vivax cases, five cases were reported negative by initial peripheral smear examination, but these cases showed scattergram abnormalities and were diagnosed as positive on repeat peripheral smear. These missed cases were found to have very low parasite index. All the P.falciparum cases were diagnosed on peripheral smear examination.Total number of smear negative cases were 2565 during this period. Both WBC-DIFF and WBC/BASO scattergrams were analyzed in all the 2610 cases. Distribution of cases is given in figure 2.

WBC-DIFF scattergram. WBC-DIFF abnormalities were found in 37 out of 45 malaria positive cases. In $P$. vivax group ( $\mathrm{n}=40) 35$ out of 40 cases showed various abnormities with a sensitivity of $87.5 \%$. In $P$. falciparum group $(\mathrm{n}=5) 2$ out of 5 cases showed WBCDIFF abnormalities with a sensitivity of $40 \%$. The overall specificity of WBC-DIFF plot in malarial detection was found to be $93.3 \%$. PPV for $P$. vivax and $P$. falciparum was $16.13 \%$ and $1.09 \%$ respectively. NPV for both groups was found to be $99.8 \%$. (Table 1)

Various changes noted in WBC-DIFF scattergram were merging of neutrophil and eosinophil clusters
(44.5\%), multiple neutrophil or eosinophil clusters (32\%), graying of neutrophil and eosinophil clusters (27\%), prominent blue coded events between/above or below the neutrophil and eosinophil clusters (22\%) and large eosinophil clusters (10\%) (Figure 3). These changes were noted singly or in combination.

WBC-DIFF abnormalities were also noted in 182 out of 2565 malaria negative cases. This included 95 new born blood samples, 46 cases of leukemia, 37 cases of other solid organ malignancies on chemotherapy and 4 cases of hemolytic anemia.

WBC/BASO scattergram. WBC/BASO channel abnormalities were found in 43 out of 45 cases. In $P$. vivax group all the cases $(\mathrm{n}=40)$ showed one single most consistent change: isolated prominent blue coded events in area III or late area II and III in the WBC/BASO scattergram with a sensitivity of $100 \%$.(Figure 4) In P. falciparum group, 3 out of 5 cases showed this change with a sensitivity of $60 \%$. Overall specificity of WBC/BASO graph in malaria detection was found to be $98.9 \%$. PPV for $P$. vivax and $P$. falciparum was $93.02 \%$ and $50 \%$ respectively.NPV for P. vivax and P. falciparum was $100 \%$ and $99.9 \%$ respectively. (Table 2)

Table 1. Sensitivity, specificity, PPV and NPV for WBC-DIFF scattergram

\begin{tabular}{|l|c|c|c|c|}
\hline & Sensitivity (\%) & Specificity (\%) & PPV (\%) & NPV (\%) \\
\hline P. vivax & 87.50 & 92.93 & 16.13 & 99.79 \\
\hline P. falciparum & 40 & 92.93 & 1.09 & 99.87 \\
\hline
\end{tabular}

Sensitivity $=$ True positive $/$ (True positive+False negative). Specificity $=$ True negative $/$ (True negative+False positive). Positive predictive value $(\mathrm{PPV})=$ True positive $/($ True positive + False positive $)$. Negative predictive value $(\mathrm{NPV})=$ True negative $/($ True negative + False negative) 

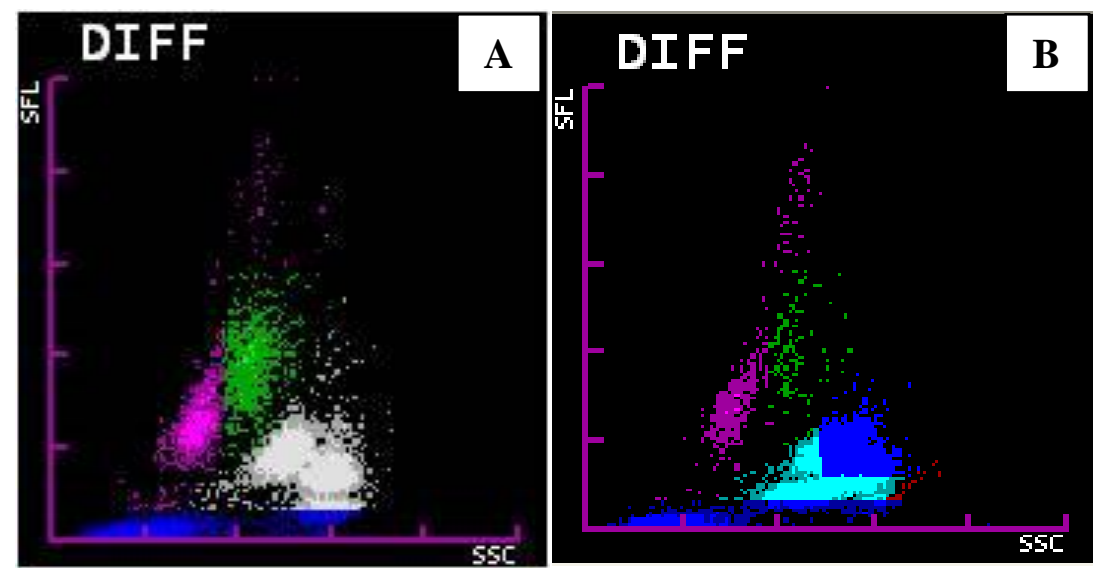

Figure 3. WBC-DIFF scattergram abnormalities. A: Graying of neutrophilic and eosinophilic clusters with blue coded events beneath. B: Merging of neutrophilic and eosinophilic clusters with blue coded events above the clusters.

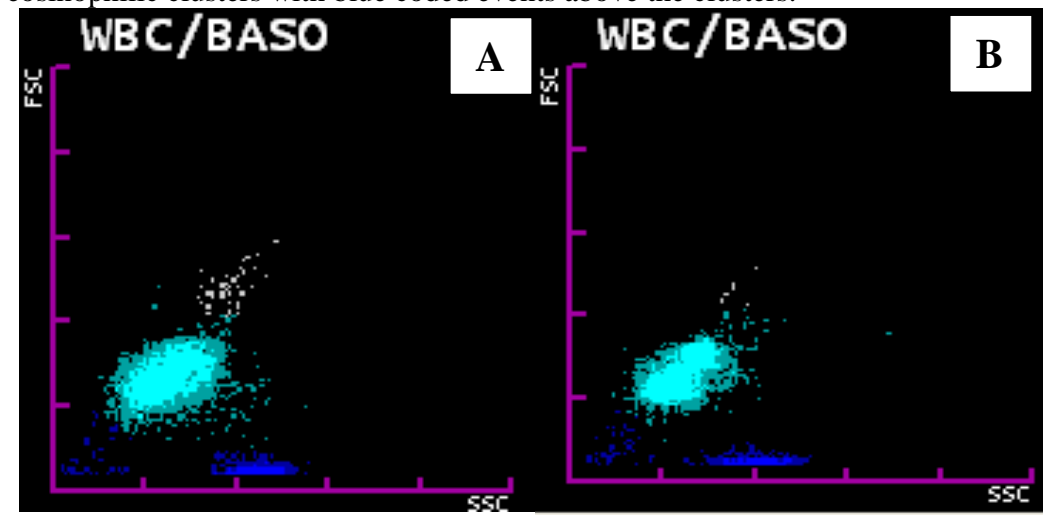

Figure 4. WBC/ BASO scattergram abnormalities. A: Showing prominent blue coded events in area III in a malaria positive case. B: Showing prominent blue coded events in late area II and area III in a malaria positive case.

Table 2. Sensitivity, specificity, PPV and NPV for WBC/BASO scattergram

\begin{tabular}{|l|c|c|c|c|}
\hline & Sensitivity (\%) & Specificity (\%) & PPV (\%) & NPV (\%) \\
\hline $\boldsymbol{P}$. vivax & 100 & 99.88 & 93.02 & 100 \\
\hline $\boldsymbol{P}$ falciparum & 60 & 99.88 & 50 & 99.92 \\
\hline
\end{tabular}

Sensitivity $=$ True positive $/$ (True positive + False negative). Specificity $=$ True negative $/$ (True negative+False positive). Positive predictive value $(\mathrm{PPV})=$ True positive / (True positive+False positive). Negative predictive value $(\mathrm{NPV})=$ True negative / $($ True negative + False negative)

Of the 5 cases of $P$. vivax which were missed on peripheral smear, fine but faint blue coded dots ranging in number from 7 to 15 were found in area III of WBC/BASO channel (Figure 5A). This gave us the opportunity to review the smears and perform rapid card tests to give a final diagnosis of malaria.

Blue coded events were noted in 3malaria negative cases out of which two cases had hemolytic disease of newborn and one was a case of thalassemia on treatment. These were confirmed to be malaria negative by a repeat smear and a negative card test. In these cases, in contrast to the malaria positive cases, the blue coding extended from area I to III (Figure 5B) Other findings. Thrombocytopenia was seen in 44out of 45 malaria positive cases with platelet count ranging from 12 - $200 \times 10^{3} / \mu 1$ with a mean of $53 \times 10^{3} / \mu 1$ (SD 31.9). Pseudoeosinophilia which is defined as a difference in automated and manual eosinophil count of $\geq 5 \%$, was noted in 7 out of 45 cases with a sensitivity of a mere $15.5 \%$. It was also noted that all the scattergram abnormalities reversed after two days of initiation of antimalarial treatment.

Discussion. In tropical and endemic countries where malaria is highly prevalent, there is a need for a rapid, cost effective and efficient method for screening the blood samples. Various new methods of malaria detection like quantitative buffy coat assay, antigen coated dipstick tests, rapid diagnostic card tests and polymerase chain reaction have come up in recent past. ${ }^{9,10}$ But these tests are limited by their high cost and limited feasibility. Due to these factors Romanowsky stained peripheral smear examination has remained as 'gold standard' in malaria diagnosis but the quality of malaria microscopy is far from satisfactory in most countries. ${ }^{11}$

There has been a growing interest in the use of automated hematology analyzers in the presumptive diagnosis of malaria. ${ }^{12}$ Earliest of such studies has been done by Hanscheid et al. ${ }^{6}$ on Cell Dyn3500 (Abott 


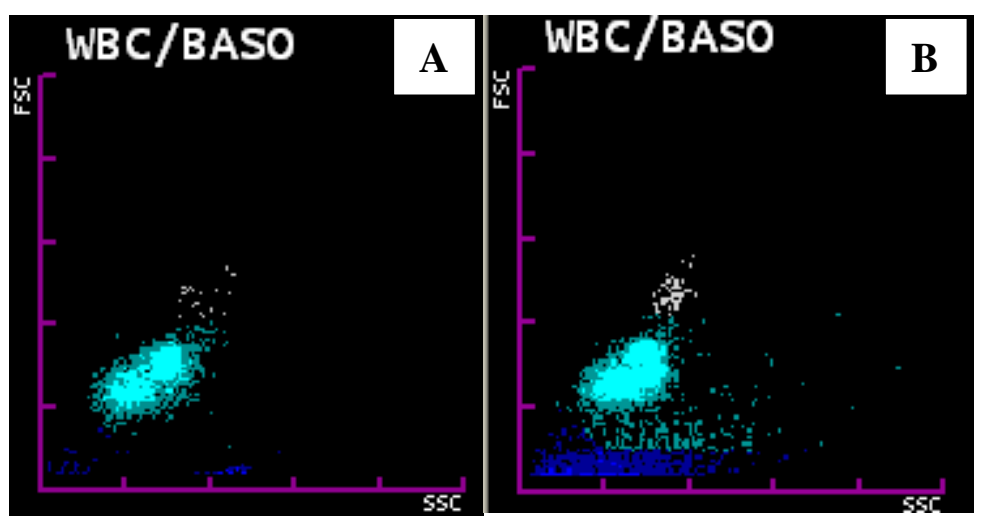

Figure 5A. WBC/ BASO scattergram showing faint blue coded events in area III in a case of P. vivax which was missed on peripheral smear examination. 5B. WBC/ BASO scattergram showing blue coded events extending from area I to III in a case of haemolytic disease of new born.

Diagnostics, USA), proving the efficacy of automated hematology analyzers in malaria detection using the principle of flow cytometry.

Sysmex XE-2100 \& XT-2000i uses flow cytometry in conjunction with fluorescence properties of leucocytes to generate various WBC parameters, a few abnormalities of which can give a clue to the diagnosis of malaria.

Zuluaga et $a l .{ }^{13}$ showed in their study that area III blue coded events in WBC/BASO scattergram for P.vivax had a sensitivity and specificity of $97 \%$ and 94\% respectively using Sysmex XE-2100 autoanalyzer. For P.falciparum the sensitivity and specificity was found to be $60 \%$ and $67 \%$ respectively. These findings were similar to that of the present study.

Also, the presence of $>8$ blue coded events in area III of WBC/BASO plot when combined with the presence of thrombocytopenia increased the sensitivity of malaria detection. These findings are similar to the findings of Zuluaga et $_{\text {al. }}{ }^{13}$

The WBC-DIFF plot abnormalities arise due to the neutrophils and eosinophils which have ingested the malarial pigment. WBC/BASO abnormalities are caused due to the red cells and reticulocytes which contain parasites and pigment. ${ }^{12}$

Yoo et $a .^{14}$ reported in their study that psuedoeosinophilia and WBC scattergram abnormalities had sensitivity of $46.2 \%$ and specificity of $99.7 \%$. Huh et al. ${ }^{15}$ also reported psuedoeosinophilia in $38 \%$ of malaria cases. In the present study psuedoeosinophilia was found in only 7 cases $(15.5 \%)$. Psuedoeosinophilia is thought to be caused by the neutrophils which contain hemozoin pigment which are erroneously plotted in the eosinophil area. Pseudoeosinophilia was not a consistent finding in our cases, in contrary to previously reported studies and this could be due to a low parasitic index/load.

We also found 3 false positive cases in the WBC/BASO scattergram, but these cases showed blue coded events extending from area I to area III in contrast to isolated area III blue coded events in malaria positive cases. In our study, WBC scattergrams showed lower sensitivity and PPV in P.falciparum detection similar to studies conducted by Jain et al.[12] and Zuluagaet al. ${ }^{13}$ This could also be attributed to the low number of $P$. falciparum cases in the current study as P.vivax is the dominant malarial parasite in this part of the country. ${ }^{16}$ However, larger studies from P.falciparum predominant areas are required to further comment on the usefulness of analyzers in diagnosis of the same. In our study, abnormalities in WBC/BASO scattergram proved to be the most consistent finding in positive cases. Moreover, 5 cases of $P$. vivax which were missed on peripheral smear showed WBC/BASO plot abnormalities.

Thrombocytopenia was present in $97.7 \%$ of malaria positive cases in our study, which was similar to the findings by Abro et al. and Chandra et al. ${ }^{17,18}$ As the number of malaria cases presenting with isolated thrombocytopenia is very high, these cases should be specifically screened for malaria infestation. ${ }^{19}$

Last but not the least, this method was found to be the most cost effective diagnostic tool as CBC analysis costs 3-4 USD when compared to Malarial card test which costs nearly 8-10 USD in our setup.

Conclusions. We found the WBC/BASO scattergram abnormalities to be useful in the presumptive diagnosis of $P$. vivax when combined with presence of thrombocytopenia. This helps the pathologists and technicians who handle these autoanalyzers to pick up all suspicious cases and subsequently confirm the same on a peripheral smear and with other rapid diagnostic tests.

Acknowledgement. Dr. K.C Mahadeva, Professor \& Head of the Department of Pathology, for his encouragement and granting permission to conduct this study. M.S Ramaiah Medical College \& Teaching Hospital. 


\section{References:}

1. Chipeta J, Mharakurwa S, Thuma P, Kumar N. A synopsis of current malaria diagnosis trends. Medical Journal of Zambia.2009; 36(2):95-101.

2. World malaria report 2013. Geneva, World Health Organization, 2013.

(http://www.who.int/malaria/publications/world_malaria_report_20 13/en/ )

3. Acharya AR, Magisetty JL, Chandra A, Chaithra BS, Khanum T, Vijayan VA. Trend of malaria incidence in the state of Karnataka, India for 2001 to 2011. Archives of applied science research.2013; 5(3):104-11.

4. Sharma S, Sethi N, Pujani M, Kushwaha S, Sehgal S. Abnormal WBC scattergram: A clue to the diagnosis of malaria. Hematol. 2012; 18(2):101-5.

http://dx.doi.org/10.1179/1607845412Y.0000000029 PMid:22980408

5. Bisoffi Z, Sirima SB, Menten J, Pattaro C, Angheben A, Gobbi F,Tinto H, Lodesani C, Neya B, Gobbo M, Van den Ende J. Accuracy of a rapid diagnostic test on the diagnosis of malaria infection and of malaria-attributable fever during low and high transmission season in Burkina Faso. Malar J. 2010; 9:192 http://dx.doi.org/10.1186/1475-2875-9-192 PMid:20609211 PMCid:PMC2914059

6. Hanscheid T, Pinto BG, Pereira I, Cristino JM, Valadas E Avoiding misdiagnosis of malaria: A novel automated method allows specific diagnosis, even in the absence of clinical suspicion. Emerg Infect Dis.1999; 5:836-38. http://dx.doi.org/10.3201/eid0506.990621 PMid:10603226 PMCid:PMC2640804

7. Inoue H. Overview of Automated Hematology Analyzer XE-2100. Sysmex Journal International.1999; 9(1):58-64

8. Ruzicka K,Veitl M,ThalhammerS,Schwarzinger,I. The New Hematology Analyzer Sysmex XE-2100:Performance Evaluation of a Novel White Blood Cell Differential Technology.Arch Pathol Lab Med.2001; 125:391-6. PMid:11231489

9. Bailey JW, Williams J, Bain BJ, William JP, Chiodin PL. Guideline: the laboratory diagnosis of malaria. British Journal of Haematology. 2013; 163: 573-80. http://dx.doi.org/10.1111/bjh.12572 PMid:24219330

10. Yan J, Li N, Wei X, Li P, Zhao Z, Wang L et al. Performance of two rapid diagnostic tests formalaria diagnosis at the ChinaMyanmar border area. Malaria Journal 2013; 12:73. http://dx.doi.org/10.1186/1475-2875-12-73
PMCid:PMC3599043

11. Bisoffi Z, Gobbi F, Buonfrate D, Ende JV. Diagnosis of Malaria Infection with or without disease. Mediterr J Hematol Infect Dis 2012; 4(1): e2012036. http://dx.doi.org/10.4084/mjhid.2012.036 PMid:22708051 PMCid:PMC3375766

12. Jain M, Gupta S, Jain J, Grover RK.Usefulness of automated cell counters in detection of malaria in a cancer set up-Our experience. IJPM. 2012; 55(4):467-73.

13. Campuzano-Zuluaga G, Alvarez-Sánchez G, Escobar-Gallo GE, Valencia- Zuluaga LM, Ríos-Orrego AM, Pabón-Vidal A, et al. Design of malaria diagnostic criteria for the Sysmex XE-2100 hematology analyzer. Am J Trop Med Hyg. 2010; 82:402-11. http://dx.doi.org/10.4269/ajtmh.2010.09-0464 PMid:20207864 PMCid:PMC2829900

14. Yoo JH, Song J, Lee KA. Short Report: Automated detection of malaria associated pseudoeosinophilia and abnormal WBCscattergramby the Sysmex-XE-2100 hematology analyzer: A clinical study with1,801 patients and real-time quantitative PCR analysis in vivax malariaendemicarea. Am J Trop Med Hyg.2010; 82:412-14. $\quad$ http://dx.doi.org/10.4269/ajtmh.2010.09-0560 PMid:20207865 PMCid:PMC2829901

15. Huh HJ, Oh GY, Huh JW, Chae SL. Malaria detection with the Sysmex XE-2100 hematology analyzer using pseudoeosinophilia and abnormal WBC scattergram. Ann Hematol. 2008; 87:755-9. http://dx.doi.org/10.1007/s00277-008-0486-8 PMid:18427808

16. Autino B, Noris A, Russo, Castelli F. Epidemiology of Malaria in Endemic Areas. Mediterr J Hematol Infect Dis 2012, 4(1): e2012060. PMid:23170189 PMCid:PMC3499992

17. Abro AH, Saleh AA, Abdou AS, Ustadi AM, Shuri M, Seliem RM. Thrombocytopenia in adults with acute malaria. RMJ. 2009; 34(2): 170-2.

18. Chandra S, Chandra H. Role of Haematological Parameters as an Indicator of Acute Malarial Infection in Uttarakhand State of India. Mediterr J Hematol Infect Dis 2013, 5(1): e2013009. http://dx.doi.org/10.4084/mjhid.2013.009 PMCid:PMC3552839

19. Saravu K, Docherla M,Vasudev A,Shastry BA. Thrombocytopenia in vivax and falciparum malaria: an observational study of 131 patients in Karnataka, India. Ann Trop Med Parasitol 2011; 105:593-598. http://dx.doi.org/10.1179/2047773211Y.0000000013 PMid:22325818 